\title{
Article \\ Unravelling the Anisotropic Behavior of Nickel-Wires Prepared through External Magnetic Field Assisted Hydrazine Reduction Method
}

\author{
Min-Soo Kim ${ }^{1,+}$, Min-Ji Shin ${ }^{2,+}$, Akshay Kumar ${ }^{2}$, Kavita Kumari ${ }^{3}$, Seok-Hwan Huh ${ }^{4}$ and Bon-Heun Koo ${ }^{5, *}$ \\ 1 School of Advanced Defense Engineering, College of Interdisciplinary Cooperative Process, Changwon \\ National University, Changwon 51140, Korea; alstn1991@changwon.ac.kr \\ 2 School of Department of Materials Convergence and System Engineering, Changwon National University, \\ Changwon 51140, Korea; tnscjfd10379@changwon.ac.kr (M.-J.S.); akshayauh@gmail.com (A.K.) \\ 3 Industrial Technology Research Institute, Changwon National University, Changwon 51140, Korea; \\ kkmalhan@gmail.com \\ 4 Department of Mechatronics Conversion Engineering, Changwon National University, \\ Changwon 51140, Korea; hsh1022@changwon.ac.kr \\ 5 School of Materials Science and Engineering, Changwon National University, Changwon 51140, Korea \\ * Correspondence: bhkoo@changwon.ac.kr \\ + First Authors: These authors contributed equally to this work.
}

check for

updates

Citation: Kim, M.-S.; Shin, M.-J.; Kumar, A.; Kumari, K.; Huh, S.-H.; Koo, B.-H. Unravelling the Anisotropic Behavior of NickelWires Prepared through External Magnetic Field Assisted Hydrazine Reduction Method. Appl. Sci. 2021, 11, 8627. https://doi.org/10.3390/ app11188627

Academic Editor: Akram Alomainy

Received: 13 August 2021

Accepted: 13 September 2021

Published: 16 September 2021

Publisher's Note: MDPI stays neutral with regard to jurisdictional claims in published maps and institutional affiliations.

Copyright: (c) 2021 by the authors. Licensee MDPI, Basel, Switzerland. This article is an open access article distributed under the terms and conditions of the Creative Commons Attribution (CC BY) license (https:/ / creativecommons.org/licenses/by/ $4.0 /)$.
Abstract: Ni wires, prepared through a hydrazine reduction, were exposed to external magnetic fields of different geometrical shape and configuration during the synthesis denoted as Ni-Non-Magnetic, Ni-Double, Ni-Single, Ni-Ring. Their effect on the wire morphology, magnetization and magnetic anisotropy was then investigated via various characterization techniques viz. X-ray diffraction (XRD), high-resolution field emission scanning electron microscopy (HR-FESEM), and vibrating sample magnetometer (VSM). The polycrystalline single phase of the Ni-wires with face centered cubic symmetry was confirmed through the analysis of XRD patterns. Analysis of HR-FESEM images revealed that the Ni-particles were aligned in form of wire-like morphology. The Ni-single sample formed the wires with minimum diameter compared to the parent sample. The magnetization measurements performed at $300 \mathrm{~K}$ and $50 \mathrm{~K}$ demonstrated the ferromagnetic behavior of all the samples. The room temperature saturation magnetization $\left(\mathrm{M}_{\mathrm{S}}\right)$ and anisotropy constant $(\mathrm{K})$ of the Ni-wires were reduced upon providing the external field during synthesis. However, the low temperature $(50 \mathrm{~K})$ magnetization behavior was rather opposing, indicating enhanced values of $\mathrm{M}_{\mathrm{S}}$ and K. Among all, Ni-ring sample showed maximum anisotropy with a value of $3.84 \times 10^{4} \mathrm{erg} / \mathrm{cm}^{3}$ at $50 \mathrm{~K}$. The ambiguous nature of the anisotropic constant and saturation magnetization ascribed partly to the variation in diameters of $\mathrm{Ni}$-wires and to the intermittent spin-spin exchange interactions of unaligned/partially aligned particles during the synthesis. Briefly, in the present study, it was established that the morphology and magnetic anisotropy of the Ni-wires could be tailored through the external magnetic field assisted synthesis method.

Keywords: Ni-wires; shape of magnetic field; magnetic anisotropy; surface anisotropy

\section{Introduction}

The one-dimensional (1D) nanomaterials have attracted much attention from the scientific community due to their functional utilization in the fabrication of optoelectronic and magnetic devices [1]. The transition-metal elements such as iron (Fe), cobalt (Co) and nickel (Ni), which have 1D nanostructures, have shown promising applications, such as in magnetic memory bits, magnetic sensors and high-density data-storage devices [2]. Their structures, possessing 1D features, exhibit unique properties, such as high aspect ratio, which can be effectively utilized for the fabrication of interconnections in devices at nanoscale. This reduces the size and weight of a device, and enhances their portability [3]. 
Among all the magnetic elements, ferromagnetic Ni is of particular interest. Due to its low magnetocrystalline anisotropy, it gives rise to enhanced magnetic properties that make it useful for the fabrication of various magnetic devices [4,5]. Along with magnetocrystalline anisotropy, 1D Ni nanostructures also exhibit a negative magnetostriction value, which makes it effective for the study of various magnetic phenomena, such as magnetization reversal, the switching behavior of magnetic fields, magnetoresistance etc. [6,7]. Magnetic anisotropy can affect the magnetic properties of a material to a great extent. For instance, A. Kumar et al. have studied the effect of magnetic anisotropy on the temperature-dependent magnetic properties of Ni nanowires and found that magnetic anisotropy increases with temperature causing increase in coercivity [7]. Similarly, the magnetotransport properties of $\mathrm{Ni}$ nanowires have also been investigated qualitatively by another research group, using an anisotropic magnetoresistance model [6,8]. Therefore, the 1D nanostructures of Ni, such as nanorods, nanobelts, nanowires etc. have been fabricated by employing various methods, such as template-assisted electrodeposition [3], electrodeposition into track-etched polymer membranes [9], chemical vapor deposition (CVD) and facile wet chemical reduction [10] etc. It is noteworthy that the control of the orientation of the easy direction in 1D structures through chemical methods is not easy; however, it can be achieved by employing various strategies [11].

In this paper, we have conducted a solution reduction experiment, and manufactured Ni wires with different shapes, with and without the application of magnetic fields, and investigated the effect of shape of the magnetic field on the magnetization and magnetocrystalline anisotropy of $\mathrm{Ni}$ wires. The advantage of applying the magnetic field during synthesis is that the easy axis of all the particles can be aligned along the wire axis. In this way, during the course of the fabrication of a wire, the magnetic field may align along the preferred crystalline orientation of the wire's axis [11]. Here, magnetic fields were applied in different geometrical shapes, such as square-type, single side and ring-type, with an intensity of 500 Gauss, as described in the Methods section. We have evaluated the structural, microstructural, and magnetic properties of nickel wires through X-ray diffraction analysis, high-resolution field emission scanning electron microscopy and DC magnetization.

\section{Materials and Methods}

The Nickel wires for the present work have been prepared using the solution synthesis method. The following required chemicals were purchased from Sigma Aldrich: $\mathrm{NiCl}_{2} \bullet 6 \mathrm{H}_{2} \mathrm{O}, \mathrm{NaOH}$ and $\mathrm{N}_{2} \mathrm{H}_{4} \bullet \mathrm{H}_{2} \mathrm{O}$ For the preparation of the wires, $\mathrm{NiCl}_{2} \bullet 6 \mathrm{H}_{2} \mathrm{O}$ was first dissolved in $20 \mathrm{~mL}$ of distilled water to make a $0.5 \mathrm{mmol}$ solution. Then, $0.04 \mathrm{~g}$ of $\mathrm{NaOH}$ was mixed with $2 \mathrm{~mL}$ of $\mathrm{N}_{2} \mathrm{H}_{4} \bullet \mathrm{H}_{2} \mathrm{O}(80 \mathrm{wt} \%)$ and distilled water to make a $0.5 \mathrm{mmol}$ transparent solution. This solution was slowly added, drop by drop, to the previously prepared $\mathrm{NiCl}_{2} \bullet 6 \mathrm{H}_{2} \mathrm{O}$ solution. Here, the color of the solution changed from green to sky blue due to a chemical reaction. The sky-blue solution is sealed at the top with a cover and heated at $120{ }^{\circ} \mathrm{C}$ for $3 \mathrm{~h}$. In the course of the experiment, the solutions gradually become transparent due to the reaction, and the black, solid product floats to the top when no magnetic field is applied (labelled as Ni-Non-Magnetic). A similar procedure was applied to prepare Ni wires in the presence of magnetic field in order to understand the effect of the magnetic field. After adding all the chemicals, as per the above-described procedure, and heating started at $120^{\circ} \mathrm{C}$, the magnetic field was applied in different shapes with the same intensity of 500 Gauss. The magnetic field was applied in various geometrical shapes: on only one side of the beaker containing the solution (labelled as Ni-Single); 250 Gauss each, on both sides of beaker and facing each other (labelled as Ni-Double); and 500 Gauss, in the shape of a ring, surrounding the entire beaker (labelled as Ni-Ring). The arrangement of the magnets to apply the magnetic field is demonstrated in the schematic shown in Figure 1. When a magnetic field was applied, the final product was accumulated at the location of the magnetic field. The resulting black solid product indicates the formation 
of Ni. The formed products were separated magnetically and washed several times with distilled water and ethanol, followed by drying at $50{ }^{\circ} \mathrm{C}$ for $5 \mathrm{~h}$ using a vacuum oven.

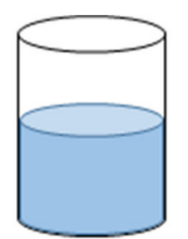

Ni-Non_Magnetic

No Applied Field

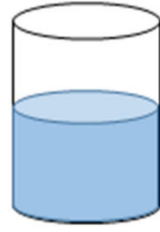

Ni_Single

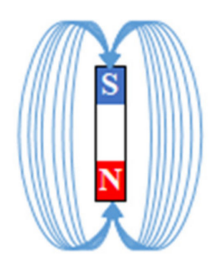

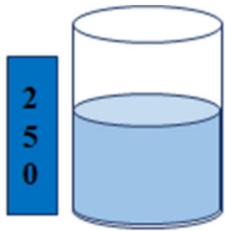

Ni_Double

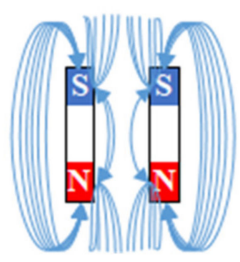

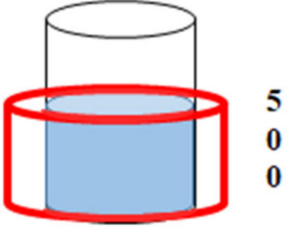

Ni_Ring

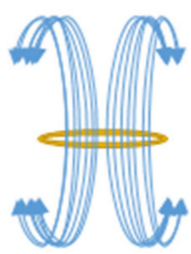

Figure 1. Schematic showing arrangement of magnets to create magnetic field with different shapes, with a magnetic field of 500 Gauss. Below the strength of the magnetic field exposed to the solution, due to the shape of the magnet, is shown.

The collected samples were characterized through XRD using X'Pert PRO MPD/Cu Ka Target $/ 40 \mathrm{Kv} / 30 \mathrm{~mA}$. High-resolution field emission scanning electron microscopy (HRFESEM) images were taken with a JSM-7900F/15 kv scanning electron, and particle size and length were analyzed and measured using Image J bundled with Java 1.8.0_172 software. The magnetization measurements were carried at the VSM module of the cryogen-free physical property measurement system (PPMS), VersaLab developed by Quantum Design.

\section{Results and Discussion}

\subsection{X-ray Diffraction Analysis}

Figure 2a represents the XRD patterns of the Ni wires synthesized in the absence and presence of external magnetic fields. The patterns show three sharp peaks, representing the polycrystalline nature of the samples. Indexing of the pattern was performed using the High Score Plus software, which assigns peaks to the (111), (200) and (220) reflections corresponding to the face-centered-cube (fcc) structure of the nickel. Peak positions correspond to the JCPDS card number 040850 [12]. The full-width-at-half maximum (FWHM), calculated from the XRD patterns, is displayed in Figure 2b, and was found to be lowest $\left(0.18^{\circ}\right)$ for the Ni-Non-Magnetic sample and highest $\left(0.48^{\circ}\right)$ for the Ni-Single sample. Further, the lattice constant was calculated using Bragg's Law. The value of the lattice constant was found to be in the range of 3.506-3.522 $\AA$, which is comparable to the reported values of the lattice constant for $\mathrm{Ni}$ in the literature. The value of the lattice parameter for fcc-Ni, reported by Mathew et al., was $3.523 \AA$ [12]. Therefore, the values of the lattice parameter found in the present case are well in agreement with the reported literature. Figure $2 c$, showing the average diameters determined using HR-FESEM images, is discussed in next section.

\subsection{Morphological Analysis}

Figure 3a-d represents the HR-FESEM images of all the samples. Figure 3a shows the wires formed in the absence of a magnetic field (Ni-Non-Magnetic sample) with relatively shorter length. A noticeable surface roughness is also observed, in these cases. The effect of an external applied magnetic field during synthesis has been observed on the length, average diameter and surface roughness of the wires. The wires suspended in solution oriented themselves along the field direction. The length of the wires increases in the samples to which a magnetic field was applied, constituted by long arrays, which can be observed in Figure $3 b-d$. Average length and average diameter were measured 
with Image J software. The average lengths of the wires were found to have increased from $28.04 \mu \mathrm{m}$, for Ni-Non-Magnetic, to $62 \mu \mathrm{m}$ for Ni-Double wires, $58 \mu \mathrm{m}$ for Ni-Single wires and $59 \mu \mathrm{m}$ for Ni-Ring wires. However, the diameter of the wires was observed to significantly decrease, by $3.0 \mu \mathrm{m}$ for Ni-Non-Magnetic wires, by $1.36 \mu \mathrm{m}$ for Ni-Double wires, by $1.28 \mu \mathrm{m}$ for Ni-Single wires and by $1.55 \mu \mathrm{m}$ for Ni-Ring samples, as displayed in Figure $2 c$ along with their standard deviations. As seen in Figure $2 b, c$, these values are in well agreements with those of FWHM calculated through XRD analysis. The lowest FWHM was found for Ni-Non-Magnetic, which led to the highest average diameter, and the highest FWHM was observed for Ni-Single wired and led to the smallest average diameter. This shows that the application of a magnetic field during synthesis helps reduce wires' diameter and enhance their length. The smallest diameter was observed for $\mathrm{Ni}$ Single wires. Nevertheless, in the cases of Ni-Double and Ni-Ring wires, the diameters of the wires were less than those observed for Ni-Non-Magnetic wires. Further, the effect of the shape of the magnetic field was observed to influence the roughness of the wires. Ni-Single wires, in addition to showing the smallest diameters, also exhibited the least roughness, as compared with the other conditions, while the greatest surface roughness was found in Ni-Ring wires. Small particles can be distinctly observed on the surface of the Ni-Ring wires, accounting for their enhanced surface roughness. It appears that the particles constituting these wires were not arranged along the axis of the wire and but rather formed protrusions in several directions, due to the ring-type arrangement of the magnet generating the magnetic field, compared to the partial effect on the solution by other magnets. Moreover, the shape of the magnetic field was also found to affect the quantity of the final product. The Ni-Ring sample produced a relatively smaller amount as compared with the other samples. Thus, the lowest diameters, longest lengths and smoothest surfaces were all found in the Ni-Single wires.

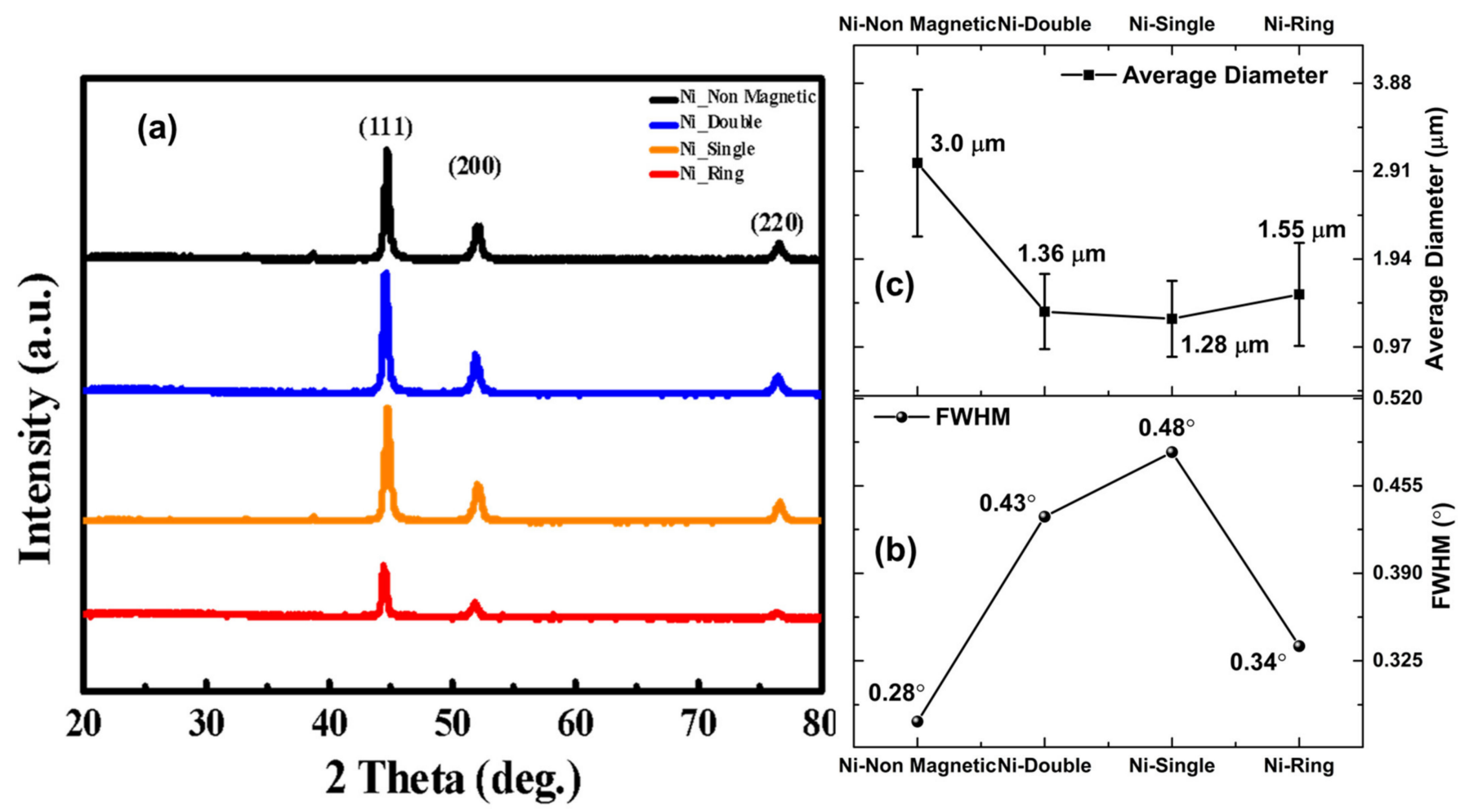

Figure 2. (a) XRD patterns of Ni-Non-Magnetic, Ni-Double, Ni-Single and Ni-Ring; (b) the values of FWHM calculated from XRD patterns; and (c) the average diameters of the Ni-wires. Error bar shows the standard deviation. 

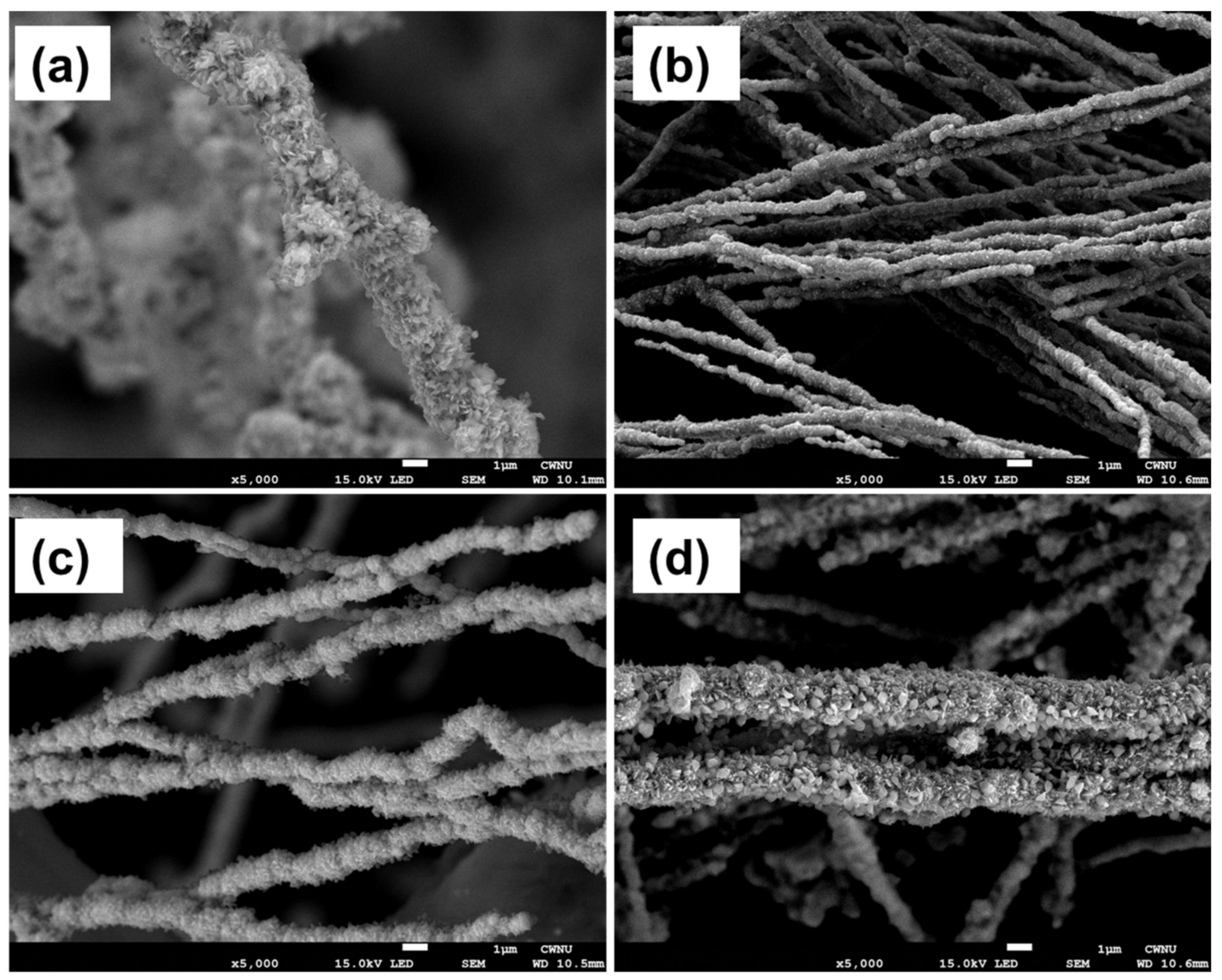

Figure 3. (a-d) SEM images (a) Ni-Non-Magnetic; (b) Ni-Single; (c) Ni-Double; (d) Ni-Ring at 5 K $\times$ magnification.

\subsection{Magnetization Analysis}

Magnetic field (H)-dependent magnetization (M) has been investigated via $\mathrm{M}-\mathrm{H}$ hysteresis curves, measured at $50 \mathrm{~K}$ and $300 \mathrm{~K}$, and are displayed in Figure 4 . All samples showed soft ferromagnetic behavior with small coercivity, as shown in the inset of Figure 4. The values of saturation magnetization (Ms) for all samples have been displayed in Table 1. The saturation magnetization, measured at $300 \mathrm{~K}$ and $50 \mathrm{~K}$, were found to be in the range 39-53 emu/g, as displayed in Table 1. These values agree with previously reported value for pure $\mathrm{Ni}, 50.62 \mathrm{emu} / \mathrm{g}$ [12]. We observed that saturation magnetization was enhanced at a lower temperature $(50 \mathrm{~K})$ as compared with room temperature $(300 \mathrm{~K})$, which is obvious. Additionally, we observed that the greatest saturation was demonstrated by Ni-Non-Magnetic wires, while it was reduced in the samples prepared with magnetic fields. Zhang et al. have also reported similar results, where Ni nanowires, assembled using external magnetic fields, exhibited lesser saturation compared with powder counterparts [2]. The reduction in the saturation magnetization of the samples prepared with magnetic fields may possibly be attributed to the shape of the magnetic field. The shape of the magnetic field mainly affects the strength of the magnetic field and the magnetic field gradient, inside the sample, and in a particular direction. It can be understood as follows: during the experiment, the sample solution was maintained between the magnets; the shapes of the magnetic fields used are been shown in Figure 1. In all the cases, the greatest magnetic field strengths were found for single magnets, and the smallest between fields generated due to two vertical magnets. 


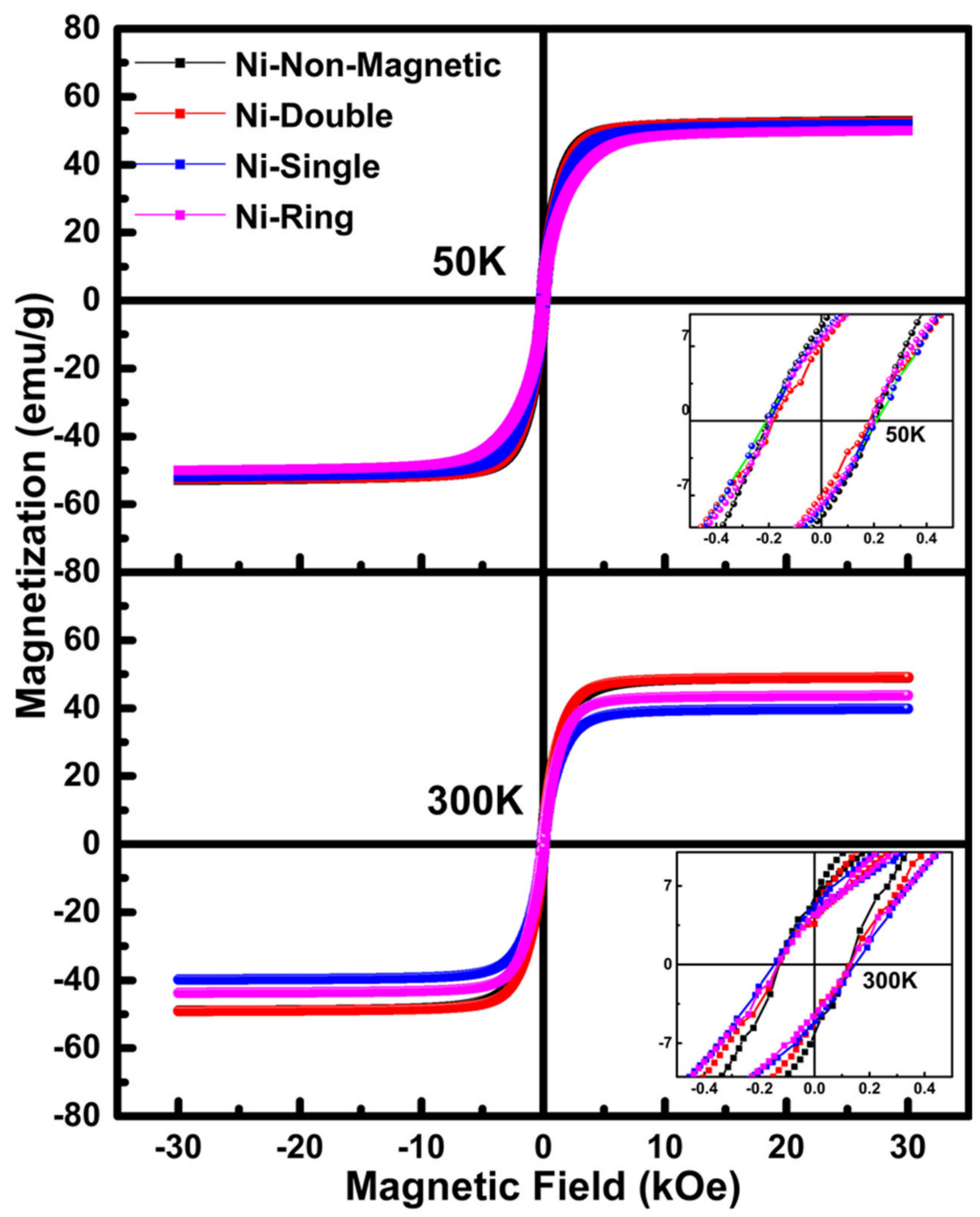

Figure 4. Room temperature magnetic hysteresis loop of Ni-Non-Magnetic, Ni-Double, Ni-Single and Ni-Ring; the inset shows hysteresis in the near $\mathrm{H}=0$ region.

Table 1. Saturation magnetization (Ms), magnetocrystalline anisotropy (K) at $300 \mathrm{~K}$ and $50 \mathrm{~K}$ temperature.

\begin{tabular}{ccccc}
\hline & \multicolumn{2}{c}{$\begin{array}{c}\text { Ms } \\
(\mathbf{e m u} / \mathbf{g})\end{array}$} & \multicolumn{2}{c}{$\begin{array}{c}\text { K }\left(\times \mathbf{1 0}^{\mathbf{4}}\right) \\
\left(\mathbf{e r g} / \mathbf{c m}^{\mathbf{3}}\right)\end{array}$} \\
\cline { 2 - 5 } & $\mathbf{3 0 0} \mathbf{K}$ & $\mathbf{5 0 ~ K}$ & $\mathbf{3 0 0 ~ K}$ & $\mathbf{5 0 ~ K}$ \\
\hline Ni-Non- & 49.1 & 52.8 & 2.78 & 2.81 \\
Magnetic & & 52.6 & 2.33 & 2.75 \\
Ni-Double & 49.0 & 51.9 & 2.15 & 3.23 \\
Ni-Single & 39.8 & 50.0 & 2.06 & 3.84 \\
Ni-Ring & 43.7 & &
\end{tabular}

In the case of Ni-Ring wires, the magnetic field strength was greatest at the center of the beaker, along the vertical axis of the ring passing through its center, and reduced towards the wall of the beaker. Therefore, the saturation magnetization observed in the Ni-Ring sample was the least. This observation indicates that the selection of the shape 
of the magnetic field during synthesis influences the saturation magnetization of the final product.

Additionally, the possibility of rotation of the direction of magnetization of individual domains in different directions cannot be declined. Due to the rotation in the direction of magnetization of individual domains, the direction of net magnetic moment directs away from the direction of easy magnetization of the metal which is $<111>$ for Ni [11]. On applying the external magnetic field during growth, the magnetic domains align in random orientations relative to the easy axis of the fcc-Ni. Similar behaviour has also been observed in earlier work [13]. Thus, during measurement, all magnetic domains are not completely aligned along the direction of easy magnetization, which thereby reduces the saturation magnetization of the samples. The lowest saturation magnetization observed in the Ni-Ring sample may be attributable to its surface roughness, which was the highest among the samples. Owing to this, the particles present on the surface contributed more to the direction of the magnetic moments pointing away from the easy magnetization direction of $\mathrm{Ni}$, and thereby reduce net saturation magnetization. In the same way, the largest saturation magnetization value (as compared with the other samples) was found for the Ni-Non-Magnetic sample, despite its surface roughness, which may be associated with the absence of an external magnetic field during synthesis [13].

Furthermore, the magnetocrystalline anisotropy present in the Ni wires was estimated using the law-of-approach-to-saturation (LAS) procedure, in stronger magnetic fields, at a temperature of $50 \mathrm{~K}$. The law-of-approach procedure can be described by the following equation $[14,15]$ :

$$
\mathrm{M}=\mathrm{Ms}\left[1-\frac{\mathbf{b}}{\mathbf{H}^{2}}\right]
$$

where $\mathrm{M}$ is the magnetic field-dependent $(\mathrm{H})$ magnetization, $\mathrm{Ms}$ is the saturation magnetization and $\mathrm{b}$ is the anisotropy term. The anisotropy constant $(\mathrm{K})$, which gives information about the strength of the magnetic anisotropy, is obtained using the value of $b$ in the following Equation (2) [14,15]:

$$
\mathrm{b}=\frac{8}{105} \frac{\mathrm{K}^{2}}{\mu_{\mathrm{o}}^{2} \mathrm{M}_{\mathrm{s}}^{2}}
$$

The law of approach fit of all the samples at temperatures of $300 \mathrm{~K}$ and $50 \mathrm{~K}$ is displayed in Figure 5. The insets of the respective figures show the enlarged portion of the fitted curve at higher magnetic fields (above 25,000 Oe). A very small separation was observed in the ranges of $\sim 0.05-0.08 \mathrm{emu} / \mathrm{g}$ at $300 \mathrm{~K}$ and $\sim 0.1-0.3 \mathrm{emu} / \mathrm{g}$ at $50 \mathrm{~K}$. The values of the anisotropy constants are displayed in Table 1 . We observed that the values of $\mathrm{K}$ increased as a consequence of a magnetic field applied externally during synthesis. The magnetic anisotropy constant provides a measurement of the anisotropic force [11]. The magnetic moments of individual particles on the surface are aligned away from the direction of easy magnetization of $\mathrm{Ni}<111>$ during the course of synthesis, therefore, significant amount of work needs to be done by the crystal increasing its magnetic anisotropy. Enhanced anisotropy in magnetically assembled nanowires has previously been reported by Tanase et al. [16]. It is noticeable that, in the present case, the maximum value of $\mathrm{K}$ was obtained for the Ni-Ring sample, which is attributable to its increased surface roughness.

Further, an increase in magnetic anisotropy can be observed in the coercivity of the samples. Figure 6 shows the increase in coercivity of the samples when the temperature was reduced from $300 \mathrm{~K}$ ( 130 Oe) to $50 \mathrm{~K}$ ( 200 Oe). Therefore, the shape of the externally applied magnetic field during synthesis contributed to the formation of Ni-wires with relatively lower diameters and enhanced surface attributes, which further modified the observed magnetic anisotropies. 

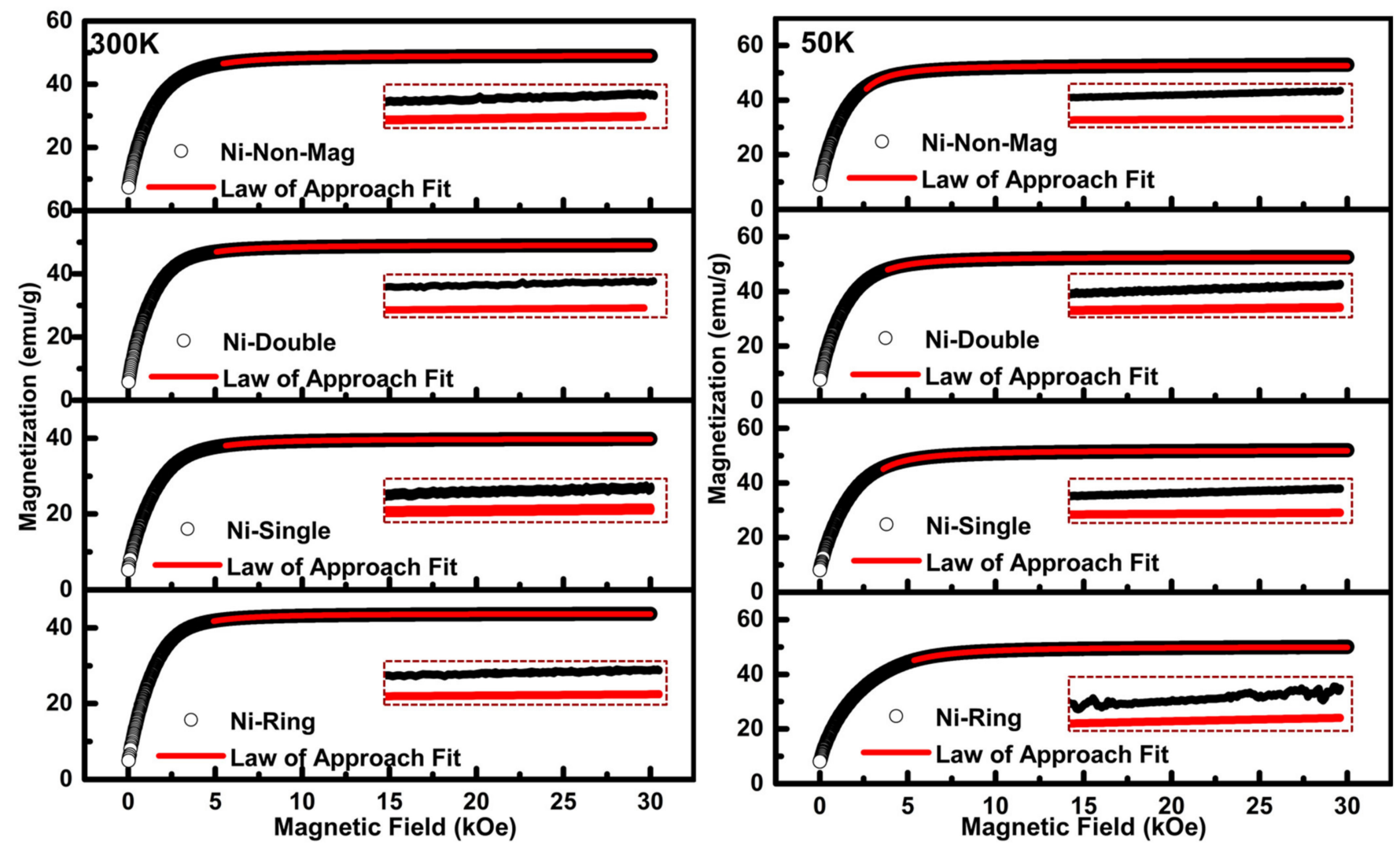

Figure 5. Law-of-approach fit of Ni-Non-Magnetic wires; Ni-Double wires; Ni-Single wires; Ni-Ring wires, using MH hysteresis curves, measured at $300 \mathrm{~K}$ and $50 \mathrm{~K}$. The insets of the respective figures show the zoom of fit of LAS at high fields ( $>25,000 \mathrm{Oe}$ ). The experimental and fitted curve show a separation in the ranges of $\sim 0.05-0.08 \mathrm{emu} / \mathrm{g}$ at $300 \mathrm{~K}$ and $\sim 0.1-0.3 \mathrm{emu} / \mathrm{g}$ at $50 \mathrm{~K}$.

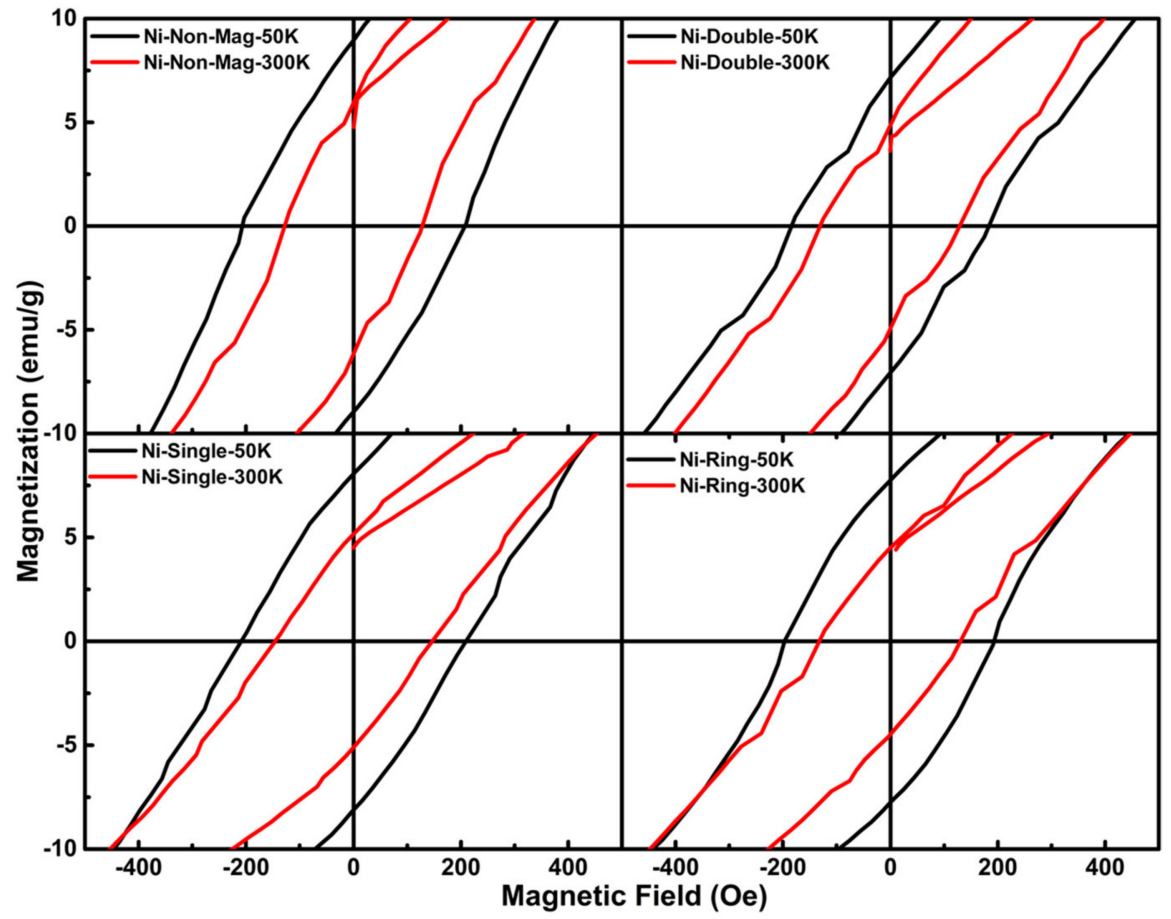

Figure 6. Comparison of MH hysteresis curves of each sample at temperatures of $300 \mathrm{~K}$ and $50 \mathrm{~K}$. 


\section{Conclusions}

The effect of the shape of tee magnetic field applied externally during the synthesis of hydrazine-reduced $\mathrm{Ni}$ wires on their magnetic properties was investigated. The prepared samples were characterized using X-ray diffraction (XRD), high-resolution field emission scanning electron microscopy (HR-FESEM), and vibrating sample magnetometer (VSM). The indexing of the XRD patterns revealed the formation of single-phase polycrystalline Ni-wires of high purity. The analysis of the HR-FESEM images showed that the Ni-Single sample produced wires of the smallest diameter $(1.28 \mu \mathrm{m})$ and enhanced surface smoothness, whereas Ni-Ring samples showed the greatest roughness. The magnetization (M) Vs magnetic field $(\mathrm{H})$ curves demonstrated the ferromagnetic nature of the samples at $300 \mathrm{~K}$ and $50 \mathrm{~K}$. Saturation magnetization and magnetic anisotropy were found to increase at lower temperatures $(50 \mathrm{~K})$. The effects of the applied magnetic fields were clearly observed on saturation magnetization and magnetic anisotropy. Saturation magnetization was found to reduce and magnetic anisotropy was found to increase in the samples prepared in the presence of a magnetic field, which may be associated with the misalignment of the magnetic moments of their surface particles away from the easy magnetization direction of $\mathrm{Ni}$.

Author Contributions: Conceptualization, B.-H.K.; methodology, S.-H.H.; software, A.K.; validation, B.-H.K.; formal analysis, B.-H.K.; investigation, M.-J.S. and M.-S.K.; resources, B.-H.K.; data curation, A.K.; writing-M.-J.S. and M.-S.K.; writing—review and editing, K.K.; visualization, B.-H.K.; supervision, B.-H.K.; project administration, B.-H.K.; funding acquisition, B.-H.K. All authors have read and agreed to the published version of the manuscript.

Funding: This research was supported by Basic Science Research Program through the National Research Foundation of Korea (NRF) funded by the Ministry of Education (No. 2020R1I1A3073553).

Institutional Review Board Statement: Not applicable.

Informed Consent Statement: Not applicable.

Data Availability Statement: Not applicable.

Acknowledgments: This research was supported by Basic Science Research Program through the National Research Foundation of Korea (NRF) funded by the Ministry of Education (No. 2020R1I1A3073553).

Conflicts of Interest: The authors declare that they have no known competing financial interest or personal relationship that could have appeared to influence the work reported in this publication.

\section{References}

1. Chang, Y.; Lye, M.L.; Zeng, H.C. Large-Scale Synthesis of High-Quality Ultralong Copper Nanowires. Langmuir 2005, 21, 3746-3748. [CrossRef] [PubMed]

2. Zhang, L.Y.; Wang, J.; Wei, L.M.; Liu, P.; Wei, H.; Zhang, Y.F. Synthesis of Ni nanowires via a hydrazine reduction route in aqueous ethanol solutions assisted by external magnetic fields. Nano-Micro Lett. 2009, 1, 49-52. [CrossRef]

3. Yoo, B.; Rheem, Y.; Beyermann, W.P.; Myung, N.V. Magnetically assembled $30 \mathrm{~nm}$ diameter nickel nanowire with ferromagnetic. Nanotechnology 2006, 17, 2512-2517. [CrossRef] [PubMed]

4. Chu, S.; Wada, K.; Inoue, S.; Todoroki, S. Fabrication and Characteristics of Ordered Ni Nanostructures on Glass by Anodization and Direct Current Electrodeposition. Chem. Mater. 2002, 14, 4595-4602. [CrossRef]

5. Vega, V.; Böhnert, T.; Martens, S.; Waleczek, M.; Montero-Moreno, J.M.; Görlitz, D.; Prida, V.M.; Nielsch, K. Tuning the magnetic anisotropy of $\mathrm{Co}-\mathrm{Ni}$ nanowires: Comparison between single nanowires and nanowire arrays in hard-anodic aluminum oxide membranes. Nanotechnology 2012, 23, 465709. [CrossRef] [PubMed]

6. Pignard, S.; Goglio, G.; Radulescu, A.; Piraux, L.; Dubois, S.; Declemy, A.; Duvail, J.L. Study of the magnetization reversal in individual nickel nanowires. J. Appl. Phys. 2000, 87, 824-849. [CrossRef]

7. Kumar, A.; Fähler, S.; Schlörb, H.; Leistner, K.; Schultz, L. Competition between shape anisotropy and magnetoelastic anisotropy in Ni nanowires electrodeposited within alumina templates. Phys. Rev. B 2006, 73, 064421. [CrossRef]

8. Wegrowe, J.; Kelly, D.; Franck, A.; Gilbert, S.E.; Ansermet, J. Magnetoresistance of Ferromagnetic Nanowires. Phys. Rev. Lett. 1999, 82, 3681-3684. [CrossRef]

9. Encinas-Oropesa, A.; Demand, M.; Piraux, L.; Huynen, I.; Ebels, U. Dipolar interactions in arrays of nickel nanowires studied by ferromagnetic resonance. Phys. Rev. B 2001, 63, 104415. [CrossRef] 
10. Kong, Y.Y.; Cem, S.; Chin, S.F. Facile synthesis of nickel nanowires with controllable morphology. Mater. Lett. $2015,142,1-3$. [CrossRef]

11. Cullity, B.D.; Graham, C.D. Introduction to Magnetic Materials; John Wiley \& Sons, Inc.: Hoboken, NJ, USA, 2008.

12. Mathew, A.; Munichandraiah, N.; Rao, G.M. Synthesis and magnetic studies of flower-like nickel nanocones. Mater. Sci. Eng. B 2009, 158, 7-12. [CrossRef]

13. Wang, J.; Chen, Q.; Zeng, C.; Hou, B. Magnetic-field-induced growth of single-crystalline $\mathrm{Fe}_{3} \mathrm{O}_{4}$ nanowires. Adv. Mater. 2004, 16, 137-140. [CrossRef]

14. Grossinger, R. A critical examination of the law of approach to saturation. I. Fit procedure. Phys. Status Solidi 1981, 66, 665-674. [CrossRef]

15. Kumar, L.; Kumar, P.; Kar, M. Cation distribution by Rietveld technique and magnetocrystalline anisotropy of Zn substituted nanocrystalline cobalt ferrite. J. Alloys Compd. 2013, 551, 72-81. [CrossRef]

16. Tanase, M.; Bauer, L.A.; Hultgren, A.; Silevitch, D.M.; Sun, L.; Reich, D.H.; Searson, P.C.; Meyer, G.J. Magnetic Alignment of Fluorescent Nanowires. Nano Lett. 2001, 1, 155-158. [CrossRef] 